CHAPTER 1

\title{
ACUTE DISSEMINATED ENCEPHALOMYELITIS
}

\author{
Joy B. Parrish* and E. Ann Yeh \\ Jacobs Neurological Institute, Department of Neurology, School of Medicine and Biomedical Sciences, University \\ at Buffalo, Buffalo, New York, USA \\ *Corresponding Author: Joy B. Parrish—Email: jparrish@thejni.org
}

\begin{abstract}
Acute disseminated encephalomyelitis (ADEM) is a disorder of the central nervous system (CNS) characterized by an acute event, typically with encephalopathy, in which diffuse CNS involvement occurs. It may follow an infectious event and occurs more commonly in young children. Pulse steroid treatment is frequently used to treat ADEM. Although ADEM is typically described as a benign condition, with children generally recovering motor function and resolution of lesions on magnetic resonance imaging (MRI), residual cognitive deficits may occur. This chapter aims to review the clinical features, typical presentation, differential diagnosis, treatment and prognosis of ADEM.
\end{abstract}

\section{INTRODUCTION}

Acute disseminated encephalomyelitis (ADEM) is a demyelinating disorder affecting the central nervous system (CNS). It typically presents in early childhood, but has been documented throughout the lifespan., ${ }^{1,2}$ According to the consensus definition of the International Pediatric MS Study Group (IPMSSG), ADEM is a "first clinical event with a presumed inflammatory or demyelinating cause, with acute or subacute onset that affects multifocal areas of the CNS". ${ }^{3}$ This definition also states that encephalopathy (behavioral change and/or alteration in consciousness) is required to make the diagnosis of ADEM. However, other literature has demonstrated that encephalopathy may be associated with a first episode of what is later diagnosed as multiple sclerosis (MS). ${ }^{4}$ Further, not all patients with ADEM present with encephalopathy. ${ }^{5}$

Neurodegenerative Diseases, edited by Shamim I. Ahmad.

(C)2012 Landes Bioscience and Springer Science+Business Media. 
Other features of ADEM included in the IPMSSG definition are: (1) neuroimaging of the brain showing focal or multifocal lesions, particularly involving white matter; (2) no history of prior demyelinating episode; (3) absence of other underlying pathology to explain the event (e.g., infection, tumor); and (4) improvement either clinically and/or on magnetic resonance imaging (MRI). The definition goes on to specify that the brain MRI may reveal large $(\geq 1-2 \mathrm{~cm})$, multifocal, hyperintense lesions in the supratentorial or infratentorial white matter regions. Gray matter may also be involved, especially the thalamus and basal ganglia and, in rare cases, there is one large lesion $(\geq 1-2 \mathrm{~cm})$ predominately involving white matter. In addition to abnormalities on brain MRI, MRI of the spinal cord may show confluent intramedullary lesions with variable enhancement. ${ }^{3}$

Although viral infection may precede symptoms of ADEM, the presence of prior infection is not required for diagnosis. Common laboratory findings include elevated white blood cell count and CSF protein. Oligoclonal bands in the CSF may be present but are less frequently found in ADEM compared to MS (see below under laboratory testing) ${ }^{6}$

Relapses have been described in children with an initial diagnosis of acute disseminated encephalomyelitis, with reported rates ranging from 5-21\%.,7-10 According to consensus definitions proposed by the IPMSSG, symptoms and/or MRI findings associated with an episode of ADEM occurring within 3 months of the initial ADEM event are considered part of the initial event. ${ }^{3}$

Recurrent ADEM (R-ADEM) is defined as the recurrence of initial symptoms and signs of the first ADEM event that occur three or more months after the initial event, without involvement of new clinical areas. The symptoms must occur at least one month after completion of steroid treatment and with no new lesions on MRI. ${ }^{3}$

Multiphasic ADEM (MDEM) is an event that meets criteria for diagnosis of ADEM but involves new areas of the CNS. Again, this event must occur three or more months after the initial event and one month following completion of steroid treatment. ${ }^{3}$ It is noted that more than two such events should raise suspicion of MS. ${ }^{3}$

These diagnostic categories were formulated in order to highlight a distinct group of patients that may experience relapses, but whose relapses will be limited to a small number and who will not progress to chronic relapses and neurodegeneration, as in MS. Although controversy regarding recurrent ADEM and MDEM exists, long term follow-up of a small group of children $(n=13)$ with relapsing forms of ADEM suggests low rates of conversion to MS (mean follow-up 9 years). In this study, only two patients who were classified as MDEM following international definitions experienced further recurrences and received alternate diagnoses (MS and CNS vasculitis). ${ }^{11}$ Further systematic evaluation of these definitions using large cohorts of patients is necessary to determine their validity.

See Table 1 for IPMSSG definitions.

\section{INCIDENCE AND PREVALENCE}

The annual incidence of ADEM is estimated to be between 0.2 and 0.8 per $100,000$. , $^{9}$-12-15 Incidence varies with geographical location, with estimates of 0.4 per 100,000 (under the age of 20 years) in California, ${ }^{9} 0.64$ per 100,000 (under the age of 15 years) in Japan, ${ }^{14} 0.07$ per 100,000 in Germany,,$^{13}$ and 0.2 per 100,000 in Canada. ${ }^{12}$ Several studies have suggested a slight predominance in males, with $\mathrm{M}$ to $\mathrm{F}$ ratios ranging from $1: 0.8$ to $2.3: 1$. $^{2,12,14-17}$ Onset usually occurs early in childhood., $.^{2,7},-9,16,18-21$ Eighty percent of patients with ADEM have onset before the age of 10 years. ${ }^{15}$ Adult onset ADEM occurs but is rare..$^{22-26}$ 
Table 1. International Pediatric MS Study Group (IPMSSG) Definitions ${ }^{3}$

\begin{tabular}{|c|c|}
\hline ADEM (monophasic) & $\begin{array}{l}\text { - First inflammatory or demyelinating clinical event } \\
\text { - Acute onset } \\
\text { - Multiple areas of the CNS affected } \\
\text { - Prysymptomatic presentation } \\
\text { in consciousness) } \\
\text { - Neuroimaging shows focal or multifocal lesions primarily } \\
\text { involving white matter } \\
\text { - No radiological evidence of previous destructive white matter } \\
\text { changes } \\
\text { - Improvement either clinically, on MRI, or both, with possible } \\
\text { residual deficits } \\
\text { - New or fluctuating symptoms within } 3 \text { months of initial event } \\
\text { considered part of the initial event } \\
\text { No other explanation for presenting symptoms }\end{array}$ \\
\hline $\begin{array}{l}\text { Recurrent ADEM } \\
\text { (R-ADEM) }\end{array}$ & $\begin{array}{l}\text { - New event of ADEM with recurrence of initial symptoms } \\
\text { occurring } 3 \text { or more months after the initial event } \\
\text { - No involvement of new areas } \\
\text { - Event occurs at least } 1 \text { month after completing steroid therapy } \\
\text { - No new lesions on MRI (there can be enlargement of original } \\
\text { lesions) }\end{array}$ \\
\hline $\begin{array}{l}\text { Multiphasic ADEM } \\
\text { (MDEM) }\end{array}$ & $\begin{array}{l}\text { - New clinical event of ADEM involving new anatomical areas } \\
\text { - Occurring at least } 3 \text { months after the initial ADEM event and at } \\
\text { least } 1 \text { month after completing steroid treatment } \\
\text { - Event must have polysymptomatic presentation including } \\
\text { encephalopathy } \\
\text { - Neurologic symptoms (other than mental status changes) must } \\
\text { differ from initial event } \\
\text { - Brain MRI must show new areas of involvement with complete } \\
\text { or partial resolution of lesions associated with the initial event } \\
\text { - More than } 2 \text { events should raise suspicion of MS }\end{array}$ \\
\hline
\end{tabular}

ADEM has been described in children who have recently experienced infections or who have received vaccinations. It is estimated that $70-77 \%$ of patients with ADEM are reported to have had clinically evident infection or vaccination during the few weeks prior to onset. ${ }^{15}$ It is important to note that ADEM rarely occurs postvaccination ( 0.1 to 0.2 cases per 100,000). ${ }^{27}$ It may occur more frequently after primary vaccination rather than revaccination. ${ }^{28}$ Case reports of ADEM after the following vaccines have been published: smallpox,,$^{28,29}$ measles, ${ }^{2,30,31}$ mumps, ${ }^{32}$ rubella, ${ }^{31}$ Japanese B encephalitis, ${ }^{33}$ diphtheria/ pertussis/tetanus, ${ }^{31}$ pertussis,,${ }^{15,31}$ influenza, ${ }^{34,35}$ hepatitis $\mathrm{B}^{19}$ and the Hog vaccine. ${ }^{36}$

Post-infectious ADEM has been described in association with viral infections including measles, ${ }^{31}$ mumps, ${ }^{2,37}$ rubella virus, ${ }^{2,31}$ varicella-zoster, ${ }^{38}$ Epstein-Barr virus, ${ }^{18,39}$ cytomegalovirus, ${ }^{40}$ herpes simplex virus, ${ }^{2,41}$ hepatitis A or $\mathrm{C},{ }^{42,43}$ Coxsackie $\mathrm{B}$ virus,${ }^{44}$ influenza $\mathrm{A}^{45}$ or $\mathrm{B},{ }^{7,18} \mathrm{H} 1 \mathrm{~N} 1$ influenza $,{ }^{46} \mathrm{HIV},{ }^{47}$ human $\mathrm{T}$-cell lymphotropic virus- $1,{ }^{48}$ human herpes virus $6,{ }^{49}$ Rocky Mountain spotted fever virus, ${ }^{50}$ and human coronavirus. ${ }^{51}$ Other pathogens include Mycoplasma pneumonia, ${ }^{52}$ Borrelia burgdorferi, ${ }^{53}$ Campylobacter, ${ }^{54}$ Leptospira, ${ }^{55,56}$ Chlamydia,${ }^{57}$ Legionella ${ }^{58}$ Rickettsia 
rickettsii,${ }^{50}$ Mycoplasma pneumonia,${ }^{52}$ Streptococcus,${ }^{59}$ and group A beta-haemolytic streptococci. ${ }^{60}$ ADEM has also been reported after organ transplantation, leukemia and non-Hodgkin's lymphoma. ${ }^{61-65}$

Systematic studies evaluating the relationship between specific infectious agents and ADEM have not been published. One study evaluating the relationship between vaccine and inflammatory demyelination has been published. Mikaeloff and colleagues reported no association between the Hepatitis B vaccination and inflammatory demyelination, although when subgroup analysis was performed on specific brands of vaccine, the Engerix B vaccine was found to increase risk, especially in children with multiple sclerosis (OR 2.77, 1.23-6.24) ${ }^{66}$ Further studies of the relationship between vaccination and inflammatory demyelination are needed to confirm the presence or absence of an association.

\section{DIFFERENTIAL DIAGNOSIS OF MULTIPLE SCLEROSIS AND ACQUIRED CENTRAL NERVOUS SYSTEM DEMYELINATING DISORDERS IN CHILDREN AND ADOLESCENTS}

The differential diagnosis of white matter abnormalities on MRI in childhood is broad. Inflammatory, infectious, metabolic and neurodegenerative disorders may present with white matter abnormalities on MRI ${ }^{67,68}$ Careful history and physical examination are necessary, but not sufficient to distinguish between children with demyelinating disorders, acute infectious or vascular processes, or chronic degenerative/metabolic processes. ${ }^{69-71}$ In many cases, MRI evaluation together with a detailed clinical history may help to distinguish metabolic white matter disorders such as Alexander's disease, Canavan's disease, Vanishing White Matter disease, Adrenoleukodystrophy and Metochromatic leukodystrophy from ADEM, particularly if attention is paid to the distribution of white matter lesions. ${ }^{72}$ Specifically, ADEM is typically acute to sub- acute in onset and causes diffuse involvement of the deep gray matter and white matter, with prominent involvement of the cerebellum in many cases. Lesions are usually large with bilateral involvement (see Fig. 1).

Importantly, the diagnosis of neuromyelitis optica (NMO) should be considered in all cases of acute demyelination in childhood, particularly in children who present with longitudinally extensive transverse myelitis and/or severe optic neuritis. Cerebral involvement with ADEM-like lesions is frequently seen in these cases; one case describes brain lesions in children who were later diagnosed with NMO. ${ }^{73}$

Even after the diagnosis of an acute demyelinating process has been established, in many cases, it is difficult to distinguish monophasic demyelinating conditions from a first attack of pediatric MS, especially in younger patients. As noted above, proposed definitions suggest that encephalopathy is required for one to diagnose ADEM, but recent reports suggest that encephalopathy may also occur at onset of neuromyelitis optica and MS, especially in younger children. ${ }^{4}$ Patients with an ADEM-like presentation whose MRI is suggestive of MS (e.g., periventricular lesions) at onset have an increased risk of developing a second episode. ${ }^{74}$

The sections below review diagnostic testing that may be of utility in distinguishing ADEM from other diseases and predicting whether children with a first presentation of ADEM will later receive the diagnosis of multiple sclerosis. 

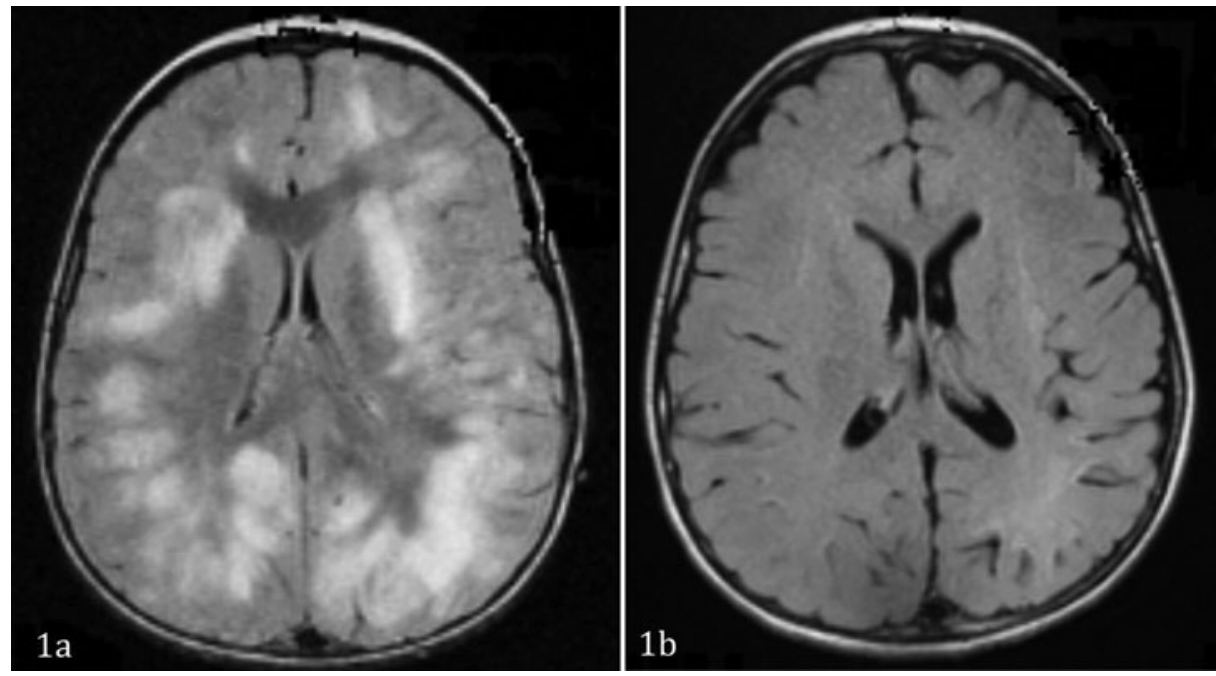

Figures 1. MRI scan of a 5 year-old child with acute disseminated encephalomyelitis at onset (1a) and 1 year later (1b). Note the diffuse involvement of both cerebral hemispheres (1a) and almost complete resolution of the previously apparent lesions (1b).

\section{DIAGNOSTIC TESTING}

Diagnostic testing for acute disseminated encephalomyelitis includes serum and CSF evaluation, visual testing and magnetic resonance imaging (MRI).

\section{Magnetic Resonance Imaging}

MRI is of central importance in making the diagnosis of ADEM. In general, as noted above, MRI lesions, associated with ADEM, include those in the deep grey nuclei, with widespread, bilateral involvement of subcortical white matter together with marked cerebellar and brainstem involvement. ${ }^{7,75}$ The spinal cord is also frequently involved. These lesions often disappear on repeated imaging.

Much work has been devoted to distinguishing ADEM, from MS. One study has suggested that the presence of well-defined periventricular and corpus callosum lesions may be specific but very insensitive predictors of MS after a first attack of CNS demyelination in childhood (KIDMUS criteria). ${ }^{74}$ More recent studies have confirmed these general findings and have found that the presence of any of the adult Barkhof criteria for MS, as well as small, well-defined lesions that are perpendicular to the corpus callosum may be effective in differentiating children with MS from those with a monophasic disease at the time of disease presentation. ${ }^{76}$ Another study reported that a combination of two of the following criteria may distinguish a first attack of pediatric MS from $\mathrm{ADEM}^{77}$ : (1) presence of T1-black holes; (2) presence of two or more periventricular lesions; and (3) absence of diffuse bilateral lesions. Importantly, this study failed to analyze the presence of gadolinium-enhancing foci that may also appear dark on T1-weighted imaging. Recently, these diagnostic criteria have been independently tested and found to be highly specific ( $95 \%$ specificity) and moderately sensitive (75\%) in distinguishing ADEM from MS. ${ }^{78}$ 


\section{CSF Analysis}

CSF analysis is central to the diagnosis and treatment of childhood demyelinating disorders. As children with ADEM often present with fever and encephalopathy, standard CSF testing is necessary, including evaluation of cell counts, protein, glucose and testing for infectious agents. Importantly, 64\% of children with ADEM are reported to have lymphocytosis on CSF analysis and $60 \%$ have CSF protein elevation. ${ }^{7}$

IgG index and oligoclonal bands (OCB) are also useful in distinguishing ADEM from MS in many cases. Up to $29 \%$ children with ADEM are reported to have oligoclonal bands in the $\mathrm{CSF},{ }^{2,6,7,19}$ while the majority of children with MS are positive for oligoclonal bands in the CSF. ${ }^{6}$ Within the French KIDMUS cohort, $94 \%$ of children with positive OCB (69/72) went on to develop MS. However, only $40 \%$ of established MS patients in this study had OCB, suggesting that this test has a low sensitivity but high specificity for the development of MS when present at disease onset. Similarly, another study reported OCB to be present in the CSF of $92 \%$ of children with MS. ${ }^{6}$

It is important to note that the CSF profile in childhood-onset demyelinating disorders may vary by age. OCB may be less frequent in younger children with MS (43\% versus $63 \%$ in adolescents). ${ }^{79}$ These results must be interpreted with caution as information regarding timing of lumbar puncture and of OCB detection was not provided in this paper, nor is it clear how many children included in this study had actually confirmed testing of OCB and/or CSF. ${ }^{21}$ The IgG index had been found to be elevated in $68 \%$ of adolescents with MS ( $>11$ years), but in $35 \%$ of younger children $(<11$ years $),{ }^{79}$ numbers similar to the rate of OCB positivity in younger children with MS and ADEM. The absence of neutrophils in the CSF at onset is predictive of an earlier second neurological episode. ${ }^{79}$

\section{Serum Testing}

No serum biomarkers have been found to be sensitive or specific for the diagnosis of ADEM. However, in a small study, children with ADEM and clinically isolated syndrome (CIS) were found to have high serum titres to nMOG (native myelin oligodendrocyte glycoprotein) in comparison to children with other neurological diseases and healthy controls. ${ }^{80}$

Serum testing may reveal leukocytosis in pediatric ADEM: almost 2/3 of patients with ADEM will present with elevated WBC as compared to $22 \%$ of children with MS. ${ }^{7}$

For children with severe optic neuritis and/or longitudinally extensive transverse myelitis, in whom the diagnosis of neuromyelitis optica is suspected, NMO IgG antibody testing should be performed. NMO-IgG testing has been found to be positive in $78 \%{ }^{73,81}$ of children who were diagnosed clinically with relapsing NMO and only $12.5 \%$ of those with monophasic NMO. ${ }^{81}$

\section{Visual Testing}

Optic neuritis may be seen in up to $60 \%$ of children presenting with ADEM, ${ }^{82}$ and almost a quarter $(23 \%)$ of children with a first time demyelinating event. ${ }^{12}$ Importantly, optic neuritis in younger children with ADEM is frequently bilateral. One study reported that $23 \%$ of children with ADEM present with bilateral optic neuritis. ${ }^{7}$ Approximately one-third of children who are later diagnosed with MS experience optic neuritis as an initial presenting symptom. ${ }^{74,83}$ Even a higher proportion of children with demyelinating 
disorders may experience subclinical abnormalities of the visual pathway. ${ }^{84}$ The limited ability of standard Snellen charts to distinguish subtle visual dysfunction is well documented in the adult MS population. ${ }^{85}$ Low contrast letter acuity charts (LCLA, Sloan charts) have been shown to provide a sensitive and reliable assessment of visual acuity in cases of pediatric demyelination. ${ }^{86}$

Other tests, such as visual evoked potential (VEP), or pattern reversal visual evoked potentials (PRVEP), have been shown to be of diagnostic utility in childhood demyelinating disorders, with almost half of such patients showing prolonged visual latency. ${ }^{84}$

More recently, optical coherence tomography (OCT), previously used for patients with glaucoma, has been applied to pediatric patients with demyelinating disorders. This procedure uses near infrared light to quantify the thickness of the retinal nerve fiber layer (RNFL) (which contains only nonmyelinated axons). It has been shown to provide a sensitive evaluation of the RNFL thickness in this population, a correlation of optic atrophy. ${ }^{86}$ Taken together, VEP, OCT and LCLA testing can provide objective evidence of previous inflammatory insult to the optic nerve in the pediatric population with demyelinating disorders. They may help to establish a diagnosis of MS and may also be used for disease monitoring on follow-up.

\section{CLINICAL AND DEMOGRAPHIC PREDICTORS OF THE RISK TO DEVELOP MS AFTER AN INITIAL DEMYELINATING EVENT}

At present, outside of MRI features, no clinical features at the time of presentation can accurately predict whether a child with an acute demyelinating event will develop MS. Clinical studies have been hampered in part by the lack of consistent definitions used across publications and the small numbers of subjects at any one site. In available studies, the risk of developing MS after ADEM has been reported to be $0 \%$ in a study from Argentina, ${ }^{2} 9.5 \%$ in a study from San Diego, ${ }^{9}$ and $18 \%$ to $29 \%$ in studies from France. ${ }^{21,74}$ Variability in the criteria used to define ADEM and pediatric MS may have contributed to the wide range of published incidence figures.

The KIDMUS study group from France examined pediatric patients with an initial demyelinating event, including CIS-like and ADEM-like events and showed that overall, $57 \%$ developed a second attack during a mean follow-up period of 5.4 years. ${ }^{21}$ Of patients presenting initially with optic neuritis, $86 \%$ developed MS, while $50 \%$ of those initially with a brainstem syndrome developed MS. Overall, positive predictive factors for the development of MS were: age at onset 10 years or older and optic nerve involvement. A lower risk of developing MS was found in patients with mental status change at presentation, suggesting that the presence of encephalopathy may be a negative predictive factor. Of patients with an initial diagnosis of ADEM, 29\% developed MS. In a subsequent publication by this group, when the diagnosis of ADEM was redefined by the KIDSEP study to include "change in mental status" as a qualifying criterion, $18 \%$ of children were found to develop MS, as defined by the development of a second event during follow-up. ${ }^{10}$

Another recent study described presenting characteristics of 89 patients who experienced an acute clinical demyelinating event, and compared those who had a monophasic course and those who ultimately experienced relapses. ${ }^{5}$ Age of onset was higher in patients with MS. Family history of MS was present in approximately $23 \%$ of MS patients and none of the ADEM cohort. Encephalopathy was present in 
approximately $41 \%$ of ADEM patients and seizure in $25 \%$ of these patients, while neither (seizure or encephalopathy) were present in any of the MS patients. Children without encephalopathy had a significantly higher likelihood of converting to MS. There was no difference between race, sex, history of preceding infection or immunization, or other features of clinical presentation. The most frequent symptom reported in MS patients was paresthesia, while weakness was most frequent in ADEM patients. CSF's white cell count and protein contents did not differ between the groups. Oligoclonal bands were positive in $8 / 18 \mathrm{MS}$ patients and none of the $\operatorname{ADEM}(\mathrm{n}=13)$ patients. IgG index was more frequently elevated in MS patients. MRI abnormalities commonly seen in MS patients included periventricular white matter lesions (generally characterized by periventricular perpendicular ovoid lesions (PVPOLs)). No ADEM patients had PVPOLs. ${ }^{5}$ Predominance of periventricular lesions in pediatric MS and relative sparing of this region in ADEM have been previously reported. ${ }^{7}$

Similar findings were reported in another study comparing pediatric MS and ADEM patients after a mean follow-up period of 5.6 years. ADEM patients more frequently experienced the following symptoms: infection prior to disease onset, polysymptomatic presentation, pyramidal signs, encephalopathy and bilateral optic neuritis $(\mathrm{ON}){ }^{7}{ }^{7}$ In this study, seizures only occurred in ADEM patients and unilateral ON occurred only in MS patients. ADEM patients were also more likely to have blood leucocytosis and nearly half had onset between the ages of 3-5 years, whereas only $23 \%$ of MS patients presented under the age of 5 years. ADEM was also more prevalent during the winter months. ${ }^{7}$

Adult onset ADEM may be characterized by a higher likelihood of relapse. One small study found that $35 \%$ of adults initially diagnosed with ADEM went on to develop MS, ${ }^{1}$ with all relapses occurring within one year of initial presentation. ${ }^{1}$ Patients eventually diagnosed with MS were more likely to have periventricular lesions ( $\mathrm{n}=14,54 \%) .{ }^{1}$ Another studies identified several factors associated with increased risk of relapse including age over 55 years, elevated CSF albumin (>100 mg/dl), female sex, spinal cord and/or PNS involvement. ${ }^{23}$

Distinguishing characteristics of monophasic ADEM in adults include preceding infection, acute onset, brainstem symptoms (e.g., ocular motor deficits, dysarthria), alteration of consciousness, aphasia, hemiplegia, paraplegia, tetraplegia, seizure, vomiting, bilateral ON, confusion, no oligoclonal bands in the CSF and gray matter involvement. ${ }^{1,25,87}$ Overall, adult patients with monophasic ADEM appear to be younger, their onset of symptoms more acute, they experience more severe initial symptoms and have more widespread CNS disturbance, but they respond more favorably to treatment. ${ }^{1}$ Information regarding ADEM in adults is limited and is based on small cohorts; therefore, definitive conclusions regarding presentation, prognosis and likelihood for relapse are limited.

See Table 2 for a description of initial presentation in ADEM versus MS in children and adolescents.

\section{TREATMENT}

\section{Steroids}

There have been no randomized, double blind studies of the treatment of ADEM. However, several retrospective analyses have suggested that high dose steroids may be used with reasonable success, ${ }^{88}$ either in the form of oral dexamethasone ${ }^{2}$ or high 
Table 2. Features of ADEM and MS in children and adolescents

\begin{tabular}{|c|c|c|}
\hline & ADEM & MS \\
\hline Age of onset & Predominantly younger (<10 yrs) & Adolescent to early adulthood \\
\hline Sex & Likely greater prevalence in males & Greater prevalence in females \\
\hline MRI & $\begin{array}{l}\text { Larger lesions; resolution of lesions } \\
\text { over time; involvement of grey and } \\
\text { white matter }\end{array}$ & $\begin{array}{l}\text { Periventricular lesions/ } \\
\text { periventricular perpendicular } \\
\text { ovoid lesions; new lesions on } \\
\text { follow-up }\end{array}$ \\
\hline CSF & $\begin{array}{l}\text { Increased WBC (neutrophils, } \\
\text { lymphocytes) }\end{array}$ & $\begin{array}{l}\text { Oligoclonal bands, elevated } \\
\text { IgG index }\end{array}$ \\
\hline Visual Testing & Bilateral ON & Unilateral ON \\
\hline Serum Testing & Leukocytosis, elevated WBC & Normal WBC \\
\hline Presentation & $\begin{array}{l}\text { Polysymptomatic, encephalopathy, } \\
\text { acute, at times severe presentation, } \\
\text { associated with infection }\end{array}$ & $\begin{array}{l}\text { Monosymptomatic, acute to } \\
\text { sub-acute onset }\end{array}$ \\
\hline
\end{tabular}

dose IV solumedrol (10-30 mg/kd/d) ${ }^{88,89}$ In many cases, the decision to intervene with steroid therapy is a clinical one. Treatment is sometimes reserved for patients with severe neurologic deficits, including visual loss, severe weakness with bowel/bladder involvement, severe encephalopathy/coma or other focal neurologic deficits, including cerebellar and brainstem deficits. One series of cases evaluating 16 patients with ADEM described response to high dose solumedrol within 10 days in $10 / 16$ patients $(63 \%) .{ }^{88}$

IVIg

IVIg therapy has been described in the treatment of ADEM, usually in the setting of steroid resistant cases ${ }^{88,90,91}$ One retrospective study examined the use of IVIg in severe steroid-resistant cases of acute disseminated encephalomyelitis. Cases received steroids as first-line therapy. A small group $(n=6)$ received IVIg as first line therapy because of contraindications for steroid use. Cases which were steroid resistant were more likely to involve peripheral nervous system damage (89\%) and myelitis $(95 \%) ; 53 \%$ of patients $(10 / 19)$ experienced clinical improvement over the course of the treatment period. ${ }^{90}$ Dosing of IVIg for acute treatment of demyelination follows other pediatric IVIg treatment protocols of $2 \mathrm{~g} / \mathrm{kg}$ total; this is frequently divided over 5 days. ${ }^{90}$

\section{Plasmapheresis}

The use of plasmapheresis for ADEM has been described in small retrospective case series of patients who are resistant to steroid therapy and/or IVIg; its benefit in ADEM has not been clearly established. One study has suggested limited recovery, which only occurred months after treatment. ${ }^{89}$ In a group of adult patients diagnosed with ADEM $(n=3)$, neurologic improvement was not seen after the administration of plasmapheresis. ${ }^{92}$ Importantly, plasmapheresis is usually reserved for the most treatment-resistant patients and is usually performed days after the onset of symptoms. It is unclear if the effect of this therapy is beneficial if given earlier in the course of the disease. 


\section{PROGNOSIS}

As mentioned earlier, the prognosis for ADEM is thought to be favorable, with more than half of patients showing complete resolution of clinical symptoms and lesions on MRI. ${ }^{2,18,60,93}$ Average time to recovery is generally over several weeks to months. ${ }^{7,18,94}$ Prognosis in adults with ADEM has been shown to be somewhat poorer than pediatric patients. ${ }^{94}$ Mortality rates are higher, ${ }^{1,24,25,87}$ up to $25 \%$, compared to less than 5\% in pediatric patients. Persistent deficits (e.g., functional state, sensory or motor disability, cranial nerve abnormality or cognitive impairment $)^{1,25,87}$ may also be more common in adult onset ADEM patients (in 35-90\%). However, differences noted between adult and pediatric ADEM populations may be due to differences in specific areas tested..$^{1,24,87}$ One study describes nearly $90 \%$ of adult patients having persistent deficits, mainly mild cognitive impairment ${ }^{87}$ studies of pediatric patients have rarely included neuropsychological data.

Over half of children diagnosed with ADEM are reported to have complete recovery. ${ }^{2,7-9,18-21}$ However, most of these studies are limited by a lack of detailed follow-up data. One study described that fewer than 17\% (3/18) of ADEM patients had continuing deficits on follow-up (2-60 months post episode). ${ }^{18}$ In this study, deficits included urinary symptoms and gait problems. A larger prospective study of 84 patients with ADEM suggested that the use of high-dose corticosteroid treatment was associated with good recovery and resolution of lesions on MRI. The majority of these patients (89\%) had either complete recovery with normal neurologic examinations or abnormal signs without disability at follow-up (mean follow-up of 6.6 years). Deficits in the remaining children included hemiparesis, partial epilepsy, decreased visual acuity, paraparesis and mental handicap. ${ }^{2}$ Within this cohort, no association could be made between initial MRI findings and outcomes. ${ }^{2}$

Another longitudinal study of 24 patients with ADEM (mean follow-up of 52.8 months) showed that only 3 had mild persistent neurologic signs. Normalization on MRI was seen in $59 \%$ of the patients and $36 \%$ showed improvement on MRI. Only one patient showed no improvement on MRI. ${ }^{93}$ A similar study including 28 pediatric ADEM patients found that $57 \%$ made a complete recovery after a mean follow-up duration of 5.8 years. Ninety percent had partial or complete resolution on MRI and no new lesions. Residual symptoms included motor impairment (17\%), parasthesia (6\%), visual impairment $(11 \%)$, cognitive impairment (11\%) and behavior problems $(11 \%){ }^{7}$

\section{Neurocognitive Functioning}

Many outcome studies in patients with ADEM fail to include assessment of cognitive and psychosocial functioning, or include only broad outcomes (e.g., IQ) and qualitative descriptions. Studies that have examined neurocognitive functioning in patients with ADEM are limited by their small sample size.

A variety of residual mild cognitive deficits in areas of visuospatial and visuomotor functioning, attention, executive function, mood, behavior and social skills have been found in children with prior diagnosis of ADEM. ${ }^{75,95-97}$ Specifically, one case-control study (ADEM $n=19$, controls $n=19$ ) showed that MRI lesion load did not correlate with cognitive performance; however, disease severity and earlier onset were associated with poorer cognitive performance. ${ }^{95}$ This study showed that early onset ADEM patients ( $<5$ years) had lower IQ and lower academic skills compared to controls, whereas older 
onset ADEM patients ( $>5$ years) had poorer verbal processing compared to controls. ${ }^{95}$ In a somewhat larger follow-up study of a group of MS, ADEM and MDEM patients (ADEM, $\mathrm{n}=28$, MDEM $\mathrm{n}=7, \mathrm{MS} \mathrm{n}=13$, with a mean follow-up 5.8 years) Dale et al (2000) found that within the ADEM/MDEM group, $11 \%$ had cognitive deficits and $11 \%$ had residual behavioral problems. Cognitive and behavioral outcomes were described as diminished IQ (IQ of 70), aggression and obsessive compulsive disorder.?

A recent study examining neurocognitive features in children with ADEM suggests mild impairment in divided attention and cognitive flexibility. ${ }^{96}$ Another small study showed ongoing cognitive deficits in all patients $(n=6)$ 2-5 years post ADEM episode, particularly in attention and executive function. This was despite complete $(\mathrm{n}=4)$ or partial resolution on MRI ${ }^{97}$ Cognitive and behavioral sequelae including low IQ, impaired speech and language, low academic achievement and aggressive behavior have also been described in a case series of pediatric ADEM patients with cerebellar involvement. ${ }^{75}$

\section{CONCLUSION}

ADEM is a rare condition with a generally favorable outcome which occurs in children, adolescents and adults. However, there is some evidence for poorer outcome in adults, with a higher risk for relapse and mortality. Approximately one-fifth of children go on to have relapses. Some of these children are eventually diagnosed with multiple sclerosis.

No single diagnostic test can reliably predict which patients with ADEM will go on to have relapses, although recently proposed MRI criteria may be helpful in this regard. Earlier detection of patients who will likely go on to have recurrent demyelination may lead to earlier treatment intervention, which may, in turn, have the potential to improve outcomes.

Finally, until recently, ADEM was assumed to be a condition with relatively few lingering deficits. However, there is growing evidence to suggest that neurocognitive impairment may persist even in the face of resolution of lesions on MRI and minimal to no lasting physical deficits. Ongoing research with larger patient samples and greater breadth in evaluation of functioning, including more sensitive measures of neurocognitive functioning, are needed.

\section{REFERENCES}

1. Schwarz S, Mohr A, Knauth M et al. Acute disseminated encephalomyelitis: A follow-up study of 40 adult patients. Neurology 2001; 56:1313-1318.

2. Tenembaum S, Cahamoles N, Fejerman N. Acute disseminated encephalomyelitis: a long-term follow-up study of 84 pediatric patients. Neurology 2002; 59(8):1224-1231.

3. Krupp LB, Banwell B, Tenembaum S. Consensus definitions proposed for pediatric multiple sclerosis and related disorders. Neurology 2007; 68(16 Suppl 2):S7-S12.

4. McKeon A, Lennon VA, Lotze T etal. CNS aquaporin-4 autoimmunity in children. Neurology 2008; 71(2):93-100.

5. Alper G, Heyman R, Wang L. Multiple sclerosis and acute disseminated encephalomyelitis diagnosed in children after long-term follow-up: Comparison of presenting features. Developmental Medicine and Child Neurology 2009; 51:480-486.

6. Pohl D, Rostasy K, Reiber H, Hanefeld F. CSF characteristics in early-onset multiple sclerosis. Neurology 2004; 63(10):1966-1967.

7. Dale RC, de Sousa C, Chong WK et al. Acute disseminated encephalomylitis, multiphasic disseminated encephalomyelitis and multiple sclerosis in children. Brain 2000; 12:2407-2422.

8. Anlar B, Basaran C, Kose G et al. Acute disseminated encephalomyelitis in children: outcome and prognosis. Neuropediatrics 2003; 34(4):194-199. 
9. Leake JA, Albani S, Kao AS et al. Acute disseminated encephalomyelitis in childhood: epidemiologic, clinical and laboratory features. Pediatr Infect Dis J 2004; 23(8):756-764.

10. Mikaeloff Y, Caridade G, Husson B et al. Acute disseminated encephalomyelitis cohort study: prognostic factors for relapse. Eur J Paediatr Neurol 2007; 11(2):90-95.

11. Olgac Dundar N, Anlar B, Guven A et al. Relapsing Acute Disseminated Encephalomyelitis in Children: Further Evaluation of the Diagnosis. J Child Neurol 2010; [Epub ahead of print]

12. Banwell B, Kennedy J, Sadovnick D et al. Incidence of acquired demyelination of the CNS in Canadian children. Neurology 2009; 72(3):232-239.

13. Tenembaum SN. Disseminated encephalomyelitis in children. Clin Neurol Neurosurg 2008; 110(9):928-938.

14. Torisu H, Kira R, Ishizaki Y et al. Clinical study of childhood acute disseminated encephalomyelitis, multiple sclerosis and acute transverse myelitis in Fukuoka Prefecture, Japan. Brain and Development 2010;32:454-462.

15. Tenembaum S, Chitnis T, Ness J et al. Acute disseminated encephalomyelitis. Neurology 2007; 68(16 Suppl 2):S23-S36.

16. Tenembaum S. Disseminated encephalomyelitis in children. Clinical Neurology and Neurosurgery 2008; 110:928-938.

17. Atzori M, Battistella PA, Perini P etal. Clinical and diagnostic aspects of multiple sclerosis and acute monophasic encephalomyelitis in pediatric patients: A single centre prospective study. Mult Sclerosis 2009; 15:363-370.

18. Murthy KSN, Faden HS, Cohen ME et al. Acute disseminated encephalomyelitis in children. Pediatrics $2002 ; 110(2)$.

19. Hynson JL, Kornberg AJ, Coleman LT et al. Clinical and neuroradiologic features of acute disseminated encephalomyelitis in children. Neurology 2001; 56(10):1308-1312.

20. Gupte G, Stonehouse M, Wassmer E et al. Acute disseminated encephalomyelitis: a review of 18 cases in childhood. J Paediatr Child Health 2003; 39(5):336-342.

21. Mikaeloff Y, Suissa S, Vallee L et al. First episode of acute CNS inflammatory demyelination in childhood: prognostic factors for multiple sclerosis and disability. J Pediatr 2004; 144(2):246-252.

22. Schwarz S, Mohr A, Knauth M et al. Acute disseminated encephalomyelitis: a follow-up study of 40 adult patients. Neurology 2001; 56(10):1313-1318.

23. Marchioni E, Tavazzi E, Minoli L etal. Acute disseminated encephalomyelitis. Neurol Sci 2008;29:S286-S288.

24. Sonneville R, Demeret S, Klein I et al. Acute disseminated encephalomyelitisin the intensive care unit:clinical features and outcome of 20 adults. Intensive Care Med 2008; 34(3):528-532.

25. Sonneville R, Klein I, de Broucker T et al. Post-infectious encephalitis in adults: diagnosis and management. J Infect 2009; 58(5):321-328.

26. Axer H, Ragoschke-Schumm A, Bottcher J et al. Initial DWI and ADC imaging may predict outcome in acute disseminated encephalomyelitis: report of two cases of brain stem encephalitis. J Neurol Neurosurg Psychiatry 2005; 76(7):996-998.

27. Menge T, Kiesseier BC, Nessler S et al. Acute disseminated encephalomyelitis: an acute hit against the brain. Curr Opin Neurol 2007; 20:247-254.

28. Booss J, Davis LE. Smallpox and smallpox vaccination: neurological implications. Neurology 2003; 60(8):1241-1245.

29. Sejvar JJ, Labutta RJ, Chapman LE et al. Neurologic adverse events associated with smallpox vaccination in the United States, 2002-2004. JAMA 2005; 294(21):2744-2750.

30. Bennetto L, Scolding N. Inflammatory/post-infectious encephalomyelitis. J Neurol Neurosurg Psychiatry 2004; (75 Suppl 1):i22-i28.

31. Fenichel GM. Neurological complications of immunization. Ann Neurol 1982; 12(2):119-128.

32. Nalin DR. Mumps, measles and rubella vaccination and encephalitis. BMJ 1989; 299(6709):1219.

33. Plesner AM, Arlien-Soborg P, Herning M. Neurological complications to vaccination against Japanese encephalitis. Eur J Neurol 1998; 5(5):479-485.

34. Cheong JH, Bak KH, Kim CHetal. Acute disseminated encephalomyelitis associated with influenza vaccination. J Korean Neurosurg Soc 2004; 35:223-225.

35. Izurieta HS, Haber P, Wise RP et al. Adverse events reported following live, cold-adapted, intranasal influenza vaccine. JAMA 2005; 294(21):2720-2725.

36. Dodick DW, Silber MH, Noseworthy JH et al. Acute disseminated encephalomyelitis after accidental injection of a hog vaccine: successful treatment with plasmapheresis. Mayo Clin Proc 1998; 73(12):1193-1195.

37. Sonmez FM, Odemis E, Ahmetoglu A et al. Brainstem encephalitis and acute disseminated encephalomyelitis following mumps. Pediatr Neurol 2004; 30(2):132-134.

38. Miller DH, Kay R, Schon F et al. Optic neuritis following chickenpox in adults. J Neurol 1986; 233(3):182-184.

39. Fujimoto H, Asaoka K, Imaizumi T et al. Epstein-Barr virus infections of the central nervous system. Intern Med 2003; 42(1):33-40.

40. Revel-Vilk S, Hurvitz H, Klar A et al. Recurrent acute disseminated encephalomyelitis associated with acute cytomegalovirus and Epstein-Barr virus infection. J Child Neurol 2000; 15(6):421-424. 
41. Kaji M, Kusuhara T, Ayabe M et al. Survey of herpes simplex virus infections of the central nervous system, including acute disseminated encephalomyelitis, in the Kyushu and Okinawa regions of Japan. Mult Scler 1996; 2(2):83-87.

42. Sacconi S, Salviati L, Merelli E. Acute disseminated encephalomyelitis associated with hepatitis C virus infection. Arch Neurol 2001; 58(10):1679-1681.

43. Tan H, Kilicaslan B, Onbas O et al. Acute disseminated encephalomyelitis following hepatitis A virus infection. Pediatr Neurol 2004; 30(3):207-209.

44. David P, Baleriaux D, Bank WO et al. MRI of acute disseminated encephalomyelitis after coxsackie B infection. J Neuroradiol 1993; 20(4):258-265.

45. Wang YH, Huang YC, Chang LY et al. Clinical characteristics of children with influenza A virus infection requiring hospitalization. J Microbiol Immunol Infect 2003; 36(2):111-116.

46. Rellosa N, Bloch KC, Shane AL et al. Neurologic Manifestations of Pediatric Novel H1n1 Influenza Infection. Pediatr Infect Dis J.

47. Silver B, McAvoy K, Mikesell S et al. Fulminating encephalopathy with perivenular demyelination and vacuolar myelopathy as the initial presentation of human immunodeficiency virus infection. Arch Neurol 1997; 54(5):647-650.

48. Tachi N, Watanabe T, Wakai S et al. Acute disseminated encephalomyelitis following HTLV-I associated myelopathy. J Neurol Sci 1992; 110(1-2):234-235.

49. Kamei A, Ichinohe S, Onuma R et al. Acute disseminated demyelination due to primary human herpesvirus-6 infection. Eur J Pediatr 1997; 156(9):709-712.

50. Wei TY, Baumann RJ. Acute disseminated encephalomyelitis after Rocky Mountain spotted fever. Pediatr Neurol 1999; 21(1):503-505.

51. Yeh EA, Collins A, Cohen ME et al. Detection of coronavirus in the central nervous system of a child with acute disseminated encephalomyelitis. Pediatrics 2004; 113(1 Pt 1):e73-e76.

52. Riedel K, Kempf VA, Bechtold A et al. Acute disseminated encephalomyelitis (ADEM) due to Mycoplasma pneumoniae infection in an adolescent. Infection 2001; 29(4):240-242.

53. van Assen S, Bosma F, Staals LM et al. Acute disseminated encephalomyelitis associated with Borrelia burgdorferi. J Neurol 2004; 251(5):626-629.

54. Nasralla CA, Pay N, Goodpasture HC et al. Postinfectious encephalopathy in a child following Campylobacter jejuni enteritis. AJNR Am J Neuroradiol 1993; 14(2):444-448.

55. Lelis SS, Fonseca LF, Xavier CC et al. Acute disseminated encephalomyelitis after leptospirosis. Pediatric Neurology 2009; 40(6):471-473.

56. Chandra SR, Kalpana D, Anilkumar TV et al. Acute disseminated encephalomyelitis following leptospirosis. J Assoc Physicians India 2004; 52:327-329.

57. Heick A, Skriver E. Chlamydia pneumoniae-associated ADEM. Eur J Neurol 2000; 7(4):435-438.

58. Spieker S, Petersen D, Rolfs A et al. Acute disseminated encephalomyelitis following Pontiac fever. Eur Neurol 1998; 40(3):169-172.

59. Dale RC, Church AJ, Cardoso F et al. Poststreptococcal acute disseminated encephalomyelitis with basal ganglia involvement and auto-reactive antibasal ganglia antibodies. Ann Neurol 2001; 50(5):588-595.

60. Huynh W, Cordato DJ, Kehdi E et al. Post-vaccination encephalomyelitis: Literature review and illustrative case. Journal of Clinical Neuroscience 2008; 15:1315-1322.

61. Au WY, Lie AK, Cheung RT et al. Acute disseminated encephalomyelitis after para-influenza infection post bone marrow transplantation. Leuk Lymphoma 2002; 43(2):455-457.

62. Horowitz MB, Comey C, Hirsch W et al. Acute disseminated encephalomyelitis (ADEM) or ADEM-like inflammatory changes in a heart-lung transplant recipient: a case report. Neuroradiology 1995;37(6):434-437.

63. Re A, Giachetti R. Acute disseminated encephalomyelitis (ADEM) after autologous peripheral blood stem cell transplant for non-Hodgkin's lymphoma. Bone Marrow Transplant 1999; 24(12):1351-1354.

64. Tomonari A, Tojo A, Adachi D et al. Acute disseminated encephalomyelitis (ADEM) after allogeneic bone marrow transplantation for acute myeloid leukemia. Ann Hematol 2003; 82(1):37-40.

65. Jaster JH, Niell HB, Dohan FC, Jr et al. Demyelination in the brain as a paraneoplastic disorder: candidates include some cases of leukemia and non-Hodgkin's lymphoma. Ann Hematol 2003; 82(11):714-715.

66. Mikaeloff Y, Caridade G, Suissa S et al. Hepatitis B vaccine and the risk of CNS inflammatory demyelination in childhood. Neurology 2008; 72(10):873-80

67. Belman A, Chabas D, Chitnis T et al. Clinical Spectrum of Disorders Masquerading as Pediatric Multiple Sclerosis. Annals of Neurology 2007; Supplement: Abstracts CNS

68. van der Knaap MS, Salomons GS, Li R et al. Unusual variants of Alexander's disease. Ann Neurol 2005; 57(3):327-338

69. Harris MO, Walsh LE, Hattab EM et al. Is it ADEM, POLG, or both? Arch Neurol 67(4):493-496.

70. Gorman MP, Golomb MR, Walsh LE et al. Steroid-responsive neurologic relapses in a child with a proteolipid protein-1 mutation. Neurology 2007; 68(16):1305-1307. 
71. Weinstock A, Giglio P, Cohen ME et al. Diffuse magnetic resonance imaging white-matter changes in a 15-year-old boy with mitochondrial encephalomyopathy. J Child Neurol 2002; 17(1):47-49.

72. van der Knaap MS, Valk J, de Neeling N et al. Pattern recognition in magnetic resonance imaging of white matter disorders in children and young adults. Neuroradiology 1991; 33(6):478-493.

73. Lotze TE, Northrop JL, Hutton GJ et al. Spectrum of pediatric neuromyelitis optica. Pediatrics 2008; 122(5):e1039-e1047.

74. Mikaeloff Y, Adamsbaum C, Husson B et al. MRI prognostic factors for relapse after acute CNS inflammatory demyelination in childhood. Brain 2004; 127(Pt 9):1942-1947.

75. Parrish JB, Weinstock-Guttman B, YehEA. Cerebellarmutism in pediatric acute disseminated encephalomyelitis. Pediatr Neurol 2010; 42(4):259-266.

76. Neuteboom RF, Boon M, Catsman Berrevoets CE et al. Prognostic factors after a first attack of inflammatory CNS demyelination in children. Neurology 2008; 71(13):967-973.

77. Callen DJ, Shroff MM, Branson HM et al. Role of MRI in the differentiation of ADEM from MS in children. Neurology 2008.

78. Ketelslegers IA, Neuteboom RF, Boon M et al. A comparison of MRI criteria for diagnosing pediatric ADEM and MS. Neurology 2010; 74(18):1412-5

79. Chabas D, Ness J, Belman A et al. Younger children with pediatric MS have a distinct CSF inflammatory profile at disease onset. Neurology 2010; 74(5):399-405.

80. Selter RC, Brilot F, Grummel V et al. Antibody responses to EBV and native MOG in pediatric inflammatory demyelinating CNS diseases. Neurology 2010; 74(21):1711-1715.

81. Banwell B, Tenembaum S, Lennon VA et al. Neuromyelitis optica-IgG in childhood inflammatory demyelinating CNS disorders. Neurology 2008; 70(5):344-352.

82. Kotlus BS, Slavin ML, Guthrie DS et al. Ophthalmologic manifestations in pediatric patients with acute disseminated encephalomyelitis. J AAPOS 2005; 9(2):179-183.

83. Simone IL, Carrara D, Tortorella C et al. Course and prognosis in early-onset MS: comparison with adult-onset forms. Neurology 2002; 59(12):1922-1928.

84. Pohl D, Rostasy K, Treiber-Held S et al. Pediatric multiple sclerosis: detection of clinically silent lesions by multimodal evoked potentials. J Pediatr 2006; 149(1):125-127.

85. Frohman E, Costello F, Zivadinov R et al. Optical coherence tomography in multiple sclerosis. Lancet Neurol 2006; 5(10):853-863.

86. Yeh E, Weinstock-Guttman B, Lincoff $\mathrm{N}$ et al. Retinal nerve fiber thickness in inflammatory demyelinating diseases of childhood onset. Mult Scler 2009; 15(7):802-810.

87. Hollinger P, Sturzenegger M, Mathis J et al. Acute disseminated encephalomyelitis in adults: a reappraisal of clinical, CSF, EEG and MRI findings. J Neurol 2001; 249:320-329.

88. Shahar E, Andraus J, Savitzki D et al. Outcome of severe encephalomyelitis in children: effect of high-dose methylprednisolone and immunoglobulins. J Child Neurol 2002; 17(11):810-814.

89. Khurana DS, Melvin JJ, Kothare SV et al. Acute disseminated encephalomyelitis in children: discordant neurologic and neuroimaging abnormalities and response to plasmapheresis. Pediatrics 2005; 116(2):431-436.

90. Ravaglia S, Piccolo G, Ceroni M et al. Severe steroid-resistant post-infectious encephalomyelitis: general features and effects of IVIg. J Neurol 2007; 254(11):1518-1523.

91. Straussberg R, Schonfeld T, Weitz R et al. Improvement of atypical acute disseminated encephalomyelitis with steroids and intravenous immunoglobulins. Pediatr Neurol 2001; 24(2):139-143.

92. Kaynar L, Altuntas F, Aydogdu I et al. Therapeutic plasma exchange in patients with neurologic diseases: retrospective multicenter study. Transfus Apher Sci 2008; 38(2):109-115.

93. Suppiej A, Vittorini R, Fontanin Metal. Acute disseminated encephalomyelitis in chidlren: Focus on Relapsing Patients. Pediatr Neurol 2008; 39:12-17.

94. Tenembaum S, Chitnis T, Ness J et al. Acute disseminated encephalomyelitis. Neurology 2007; 68(Suppl 2):S23-S36.

95. Jacobs RK, Anderson VA, Neale JL et al. Neuropsychological outcome after acute disseminated encephalomyelitis: impact of age at illness onset. Pediatr Neurol 2004; 31(3):191-197.

96. Deery B, Anderson V, Jacobs Retal. Childhood MS and ADEM: Investigation and comparison of neurocognitive features in children. Developmental Neuropsychology 2010; 35(5):506-521.

97. Hahn CD, Miles BS, MacGregor DL et al. Neurocognitive outcome after acute disseminated encephalomyelitis. Pediatr Neurol 2003; 29:117-123. 